\section{Synthesis and Antibacterial Activities of Novel C(7)-Catechol-substituted Cephalosporins (I)}

\author{
Yong-Zu KIM*, Jong-Chan Lim, JAE-Hong YeO, \\ Chan-Sik Bang, Sam-Sik Kim, Tae Hee Lee, \\ Seong Ho OH, Young-Choon MoOn \\ and CHANG-SEOK LEE
}

Biotech Research Institute, Research Park, LG Chemical Ltd.,

P.O. Box 61Yu sung. Science Town,

Taejon 305-380, Republic of Korea

(Received for publication February 2, 1996)

Cephalosporins have been widely used for treating diseases caused by pathogenic bacteria in human and animals. Through the ton $B$ dependent iron transport mechanism, ${ }^{1,2)}$ the use of catechol substituted $\beta$-lactams was known to increase the drug's penetration into the bacterial cell walls. Thus, several catechol-substituted $\beta$-lactams have been synthesized and showed good antipseudomonal activities. ${ }^{3 \sim 8)}$ Despite their excellent antipseudomonal activities compared with those of other cephalosporins, most of the catechol substituted cephalosporins have weak activity against Gram-positive bacteria. ${ }^{3 \sim 73}$ Also, there has been emerging resistance against cephalosporins, such as resistant Escherichia coli expressing TEM-like $\beta$-lactamases.

In the previous paper, ${ }^{9)}$ a new class of cephalosporins bearing 3-[(aminopyrimidiniumyl)thio] methyl substituents was found to exhibit well balanced activities against Gram-positive and Gram-negative bacteria. By introducing the cell penetration mechanism of the above catechol substituents on cephalosporins, while maintaining 3-[(aminopyrimidiniumyl)thio] methyl substituents on cephem nucleus, we wish to synthesize new cephalosporins which have not only broader spectrum of antibacterial activities including Pseudomonas aeruginosa, but also increased stabilities against various $\beta$-lactamases and longer plasma half life. Thus, we have prepared a series of novel compounds which possess both catechol moiety at the 7-position and pyrimidiniumthio methyl, pyrimidinothio methyl or pyrimidinium methyl group at the 3-position of cephem nucleus (Fig. 1). We report herein the synthesis of these compounds and their antimicrobial activities.

\section{Chemistry}

The compounds $1 \sim 10$ from oxime 11 and bromide 12 were prepared as follows (Scheme 1). Oxime 11 and benzyl bromide 12 was coupled in the presence of potassium carbonate and potassium iodide in $N, N$ dimethylformamide (DMF) to afford the oxyimino compound 13. Removal of the allyl moiety on the compound 13 was performed by using $\operatorname{Pd}(0)$ catalyzed reaction to give the carboxylic acid 14 . The acid 14 was added to the methylene chloride solution containing p-methoxybenzyl-7-amino-3-chloromethyl-3-cephem-4carboxylate (7-ACLE) and pyridine at $-20^{\circ} \mathrm{C}$, then phosphorous oxychloride $\left(\mathrm{POCl}_{3}\right)$ was added to the solution to afford the coupling product 15 . Then the chloride 15 was displaced with pyrimidine thiones or pyrimidines in DMF and deprotection of the corresponding coupling products in the presence of trifluoroacetic acid (TFA) and anisole afforded the cephalosporins $1 S, R \sim 10 S, R^{\dagger}$ (Most compounds have two diastereomers which contain $R$ and $S$ configuration on the benzylic position of catechol moiety. Thus, from now on, each diastereomers will be described as $R$ and $S$ ). Spectra for 1S: IR (nujol) $1770 \mathrm{~cm}^{-1}$ (carbonyl on $\beta$-lactam ring); ${ }^{1} \mathrm{H}$ NMR $\left(\delta, \mathrm{D}_{2} \mathrm{O}\right) 3.29(\mathrm{ABq}, 2 \mathrm{H}, J=15.1 \mathrm{~Hz}), 4.07$ $(\mathrm{ABq}, 2 \mathrm{H}, J=13.5 \mathrm{~Hz}), 4.96(\mathrm{~d}, 1 \mathrm{H}, J=2.5 \mathrm{~Hz}), 5.37(\mathrm{~s}$, $1 \mathrm{H}), 5.42(\mathrm{~s}, 1 \mathrm{H}), 5.62(\mathrm{~d}, 1 \mathrm{H}, J=2.5 \mathrm{~Hz}), 6.81(\mathrm{~d}, 1 \mathrm{H}$, $J=7.8 \mathrm{~Hz}), 6.91(\mathrm{~d}, 1 \mathrm{H}, J=7.8 \mathrm{~Hz}), 7.01(\mathrm{~s}, 2 \mathrm{H})$.

Antibacterial Activities and Discussion

Agar dilution method was used to determine the minimal inhibitory concentration (MIC) of compounds $1 \sim 10$ against selected organisms. The MIC values for ceftazidime against the same strains are shown for comparison. In general, most of the compounds in Table 1 showed better antibacterial activities than those of the

Fig. 1. Novel catechol-substituted cephalosporins $1 \sim 10$ with various $\mathrm{C}-3$ substituents.
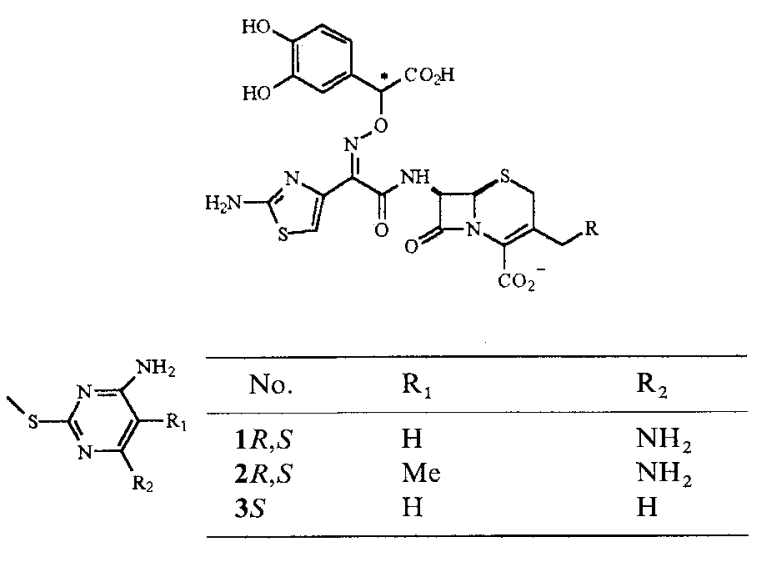

\begin{tabular}{llll}
\cline { 2 - 3 } & \multicolumn{2}{c}{$\mathrm{R}_{1}$} & $\mathrm{R}_{2}$ \\
\cline { 2 - 4 } & $\begin{array}{l}\mathrm{No} \\
4 R, S\end{array}$ & $\mathrm{H}$ \\
$5 R, S$ & $-\mathrm{CH}_{2} \mathrm{CH}_{2} \mathrm{CH}_{2}-$ & $\mathrm{H}$ \\
\hline
\end{tabular}

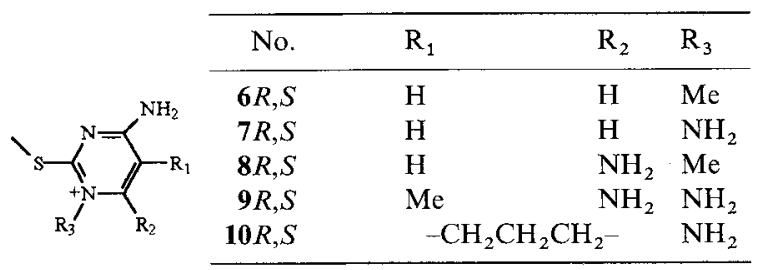

\footnotetext{
$R$ and $S$ configurations were proved by the method shown in the ref. 10 .
} 
Scheme 1.
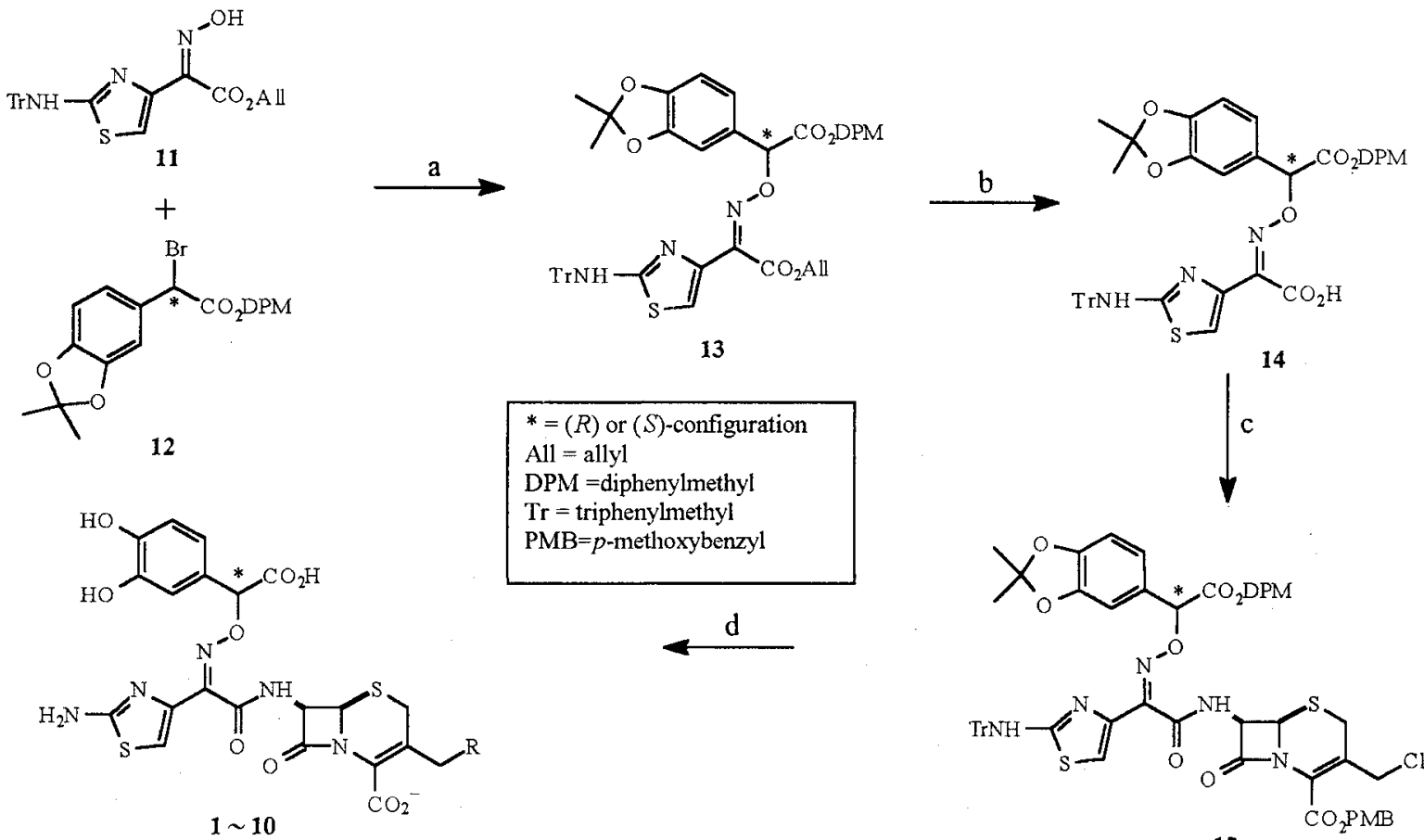

a) $\mathrm{K}_{2} \mathrm{CO}_{3}, \mathrm{KI}$, DMF; b) $\mathrm{Pd}\left(\mathrm{PPh}_{3}\right)_{4}$, potassium 2-ethylhexanoate; c) $\mathrm{POCl}_{3}$, 7-ACLE; d) (1)Nucleophile (R); (2) TFA, Anisole.

Table 1. Antibacterial activities of cephalosporins $1 \sim 10(\mathrm{MIC}, \mu \mathrm{g} / \mathrm{ml})$.

\begin{tabular}{llrllllrll}
\hline Compound & S.a. & $E . f$. & $E . c .1$ & $E . c .2$ & P.a. & A.c. & E.c. & K.a. & S.m. \\
\hline $\mathbf{1 S}$ & 2 & $>128$ & $<0.008$ & 0.031 & 0.25 & 0.13 & 16 & 0.13 & 0.25 \\
$\mathbf{2} S$ & 2 & $>128$ & $<0.008$ & 0.031 & 0.25 & 0.5 & 8 & 0.13 & 0.25 \\
$\mathbf{3} S$ & 4 & $>128$ & 0.063 & 0.13 & 1 & 2 & 128 & 0.5 & 1 \\
$\mathbf{4} S$ & 0.5 & 32 & 0.13 & 0.13 & 2 & 1 & 64 & 1 & 0.5 \\
$\mathbf{5 S}$ & 0.5 & 4 & 0.063 & 0.13 & 1 & 1 & 16 & 1 & 0.25 \\
$\mathbf{6 S}$ & 0.5 & 64 & 0.031 & 0.063 & 0.5 & 0.5 & 16 & 0.25 & 0.5 \\
$\mathbf{7 S}$ & 1 & 64 & 0.016 & 0.063 & 0.25 & 0.25 & 8 & 0.13 & 0.25 \\
$\mathbf{8} S$ & 0.5 & 32 & 0.063 & 0.063 & 0.5 & 0.5 & 16 & 0.25 & 0.5 \\
$\mathbf{9 S}$ & 0.25 & 32 & 0.031 & 0.063 & 0.5 & 0.5 & 8 & 0.13 & 0.25 \\
$\mathbf{1 0} S$ & 0.5 & 16 & 0.031 & 0.063 & 0.5 & 0.25 & 2 & 0.13 & 0.5 \\
Ceftazidime & 16 & $>128$ & 0.13 & 0.25 & 1 & 2 & 64 & 0.25 & 0.25 \\
\hline
\end{tabular}

S.a., Staphylococcus aureus ATCC-6538p; E.f., Enterococcus faecalis 29212; E.c.1, Escherichia coli ATCC-10536; E.c.2, Escherichia coli TEM1 1193E; P.a., Pseudomonas aeruginosa 1912E; A.c., Acinetobacter calcoaceticus 15473; E.c., Enterobacter cloacae P99; K.a., Klebsiella aerogenes SHV-1 1976E; S.m., Serratia marcescens 1826E.

Table 2. Pharmacokinetic data of the compounds $1 \sim 4,6 \sim 10$ in rats.

\begin{tabular}{lrrrrrrrrrr}
\hline \multicolumn{1}{c}{ Parameters } & \multicolumn{1}{c}{$\mathbf{1 S}$} & \multicolumn{1}{c}{$\mathbf{2 S}$} & $\mathbf{3 S}$ & $\mathbf{4 S}$ & $\mathbf{6 S}$ & $\mathbf{7 S}$ & $\mathbf{8 S}$ & $\mathbf{9 S}$ & $\mathbf{1 0 S}$ & $\mathrm{CAZ}$ \\
\hline $\mathrm{T}_{1 / 2}($ minute $)$ & 62 & 55 & 58 & 59 & 39 & 50 & 53 & 40 & 53 & 20 \\
$\mathrm{AUC}(\mu \mathrm{g} \cdot$ minute $/ \mathrm{ml})$ & 3694 & 3247 & 3472 & 3571 & 2614 & 3174 & 3324 & 3152 & 3868 & 1863 \\
\hline
\end{tabular}


reference (CAZ: ceftazidime). This series of new catechol substituted cephalosporins exhibited good antibacterial activities against Gram-positive bacteria such as $S$. aureus and excellent activities against Gram-negative organisms including Pseudomonas aeruginosa. The cephalosporins $1 \sim 10$ showed better antibacterial activities against most of the Gram-positive strains than the other catecholcontaining cephalosporins. ${ }^{3 \sim 8)}$ Interestingly, most cephalosporins shown in Table 1 displayed poor activity against $E$. faecalis. It is worthwhile to note that the compounds having $(S)$-configuration on the catechol side chain were significantly more active than the compound which contains the corresponding $(R)$-side chain (The MIC's of $(R)$-isomers are not shown in this paper but in paptents). ${ }^{11)}$ Cephalosporins with pyrimidiniumyl substituents 4, 5 exhibited similar potency against Gram positive bacteria to the compounds substituted by thiopyrimidiniumyl $\mathbf{6} \sim \mathbf{1 0}$ and thiopyrimidinyl derivatives $1 \sim 3$, but showed poor activity against Gram-negative strains, especially against $P$. aeruginosa. Among these series of compounds, cephalosporins with thiopyrimidiniumyl moiety exhibited the most balanced antibacterial activity profiles.

As expected by the mechanism described before, cephalosporins $\mathbf{1} \sim \mathbf{1 0}$ had a good potency against $P$. aeruginosa, especially the $(S)$-configurated diastereomers showed excellent anti-pseudomonal activities. The compounds $1 \sim \mathbf{1 0}$ were also very stable to the extended spectrum of TEM-like $\beta$-lactamases. Thus, they possessed very good activities against resistant $E$. coli which express TEM $1 \beta$-lactamases. They also displayed better antibacterial activities against $K$. aerogenes expressing SHV-1 $\beta$-lactamase than that of the ceftazidime, but gave comparable activities against $E$. cloaceae expressing P99 $\beta$-lactamase compared to CAZ. Pharmacokinetic studies on the new cephalosporins $\mathbf{1} \sim \mathbf{3}, \mathbf{6}, \mathbf{7} \sim \mathbf{1 0}$ which have pyrimidinyl- and pyrimidinium-thiomethyl substituents were shown in Table 2 . In rats, they showed significantly higher AUC values and longer half life compared to ceftazidime after a dose of $20 \mathrm{mg} / \mathrm{kg}$ intravenously.

\section{References}

1) Silley, P.; J. W. Griffiths, D. Monsey \& A. M. Harris: Mode of action of GR69153, a novel catechol-substituted cephalosporin, and its interaction with the ton $B$ dependent iron transport system. Antimicrob. Agents Chemother. 34: $1806 \sim 1808,1990$

2) NeILANDs, J. B.: Microbial envelope proteins related to iron. Annu. Rev. Microbiol. 36: 285 309, 1982

3) Erwin, M. E.; R. N. Jones, B. M. Barrette \& D. M. JoHNSON: In vitro evaluation of GR69153, a novel catechol-substituted cephalosporin. Antimicrob. Agents Chemother. 35: 929 937, 1991

4) Watanabe, N. A.; T. Nagasu, K. Katsu \& K. Kitoh: E-0702, a new cephalosporin, is incorporated into $E$. coli cells via the ton $B$ dependent iron transport mechanism. Antimicrob. Agents Chemother. 31: $497 \sim 504,1987$

5) JONES, R. N. \& M. E. ERwiN: In vitro evaluation of Ro 09-1227, a novel catechol-substituted cephalosporin. Antimicrob. Agents Chemother. 36: 233 238, 1992

6) Mochizuki, H.; H. Yamada, Y. OiKawa, K. Murakami, J. Ishiguro, H. Kosuzume, N. Arizawa \& E. Mochida: Bactericidal activity of M14659 enhanced in low-iron environments. Antimicrob. Agents Chemother. 32: 1648 1654,1988

7) NaKagawa, S.; M. Sanada, K. Matsuda, T. Hashizume, Y. Asahi, R. Ushimma, N. OHTAKe \& N. TANAKA: In vitro and in vivo antibacterial activities of BO-1341, a new antipseudomonal cephalosporin. Antimicrob. Agents Chemother. 33: 1423 1427, 1989

8) Arnould, J. C.; A. Bertrandie, T. G. C. Bird, D. Boucherot, F. Jung, J. J. Lohmann, A. Oliver, J. P. Bailey, W. Bell \& G. M. Davies: Synthesis and structure-activity relationship of cephalosporins with C-3' catechol-containing residues. J. Med. Chem. 35: $2631 \sim$ 2642, 1992

9) Kim, Y.-Z.; J.-C. LiM, J.-H. YeO, C.-S. BANG, W.-S. KiM, S.-S. KIM, Y.-M. WoO, D.-H. YanG, H. S. OH \& K. NAHM: Synthesis and antimicrobial activity of novel 3-[(aminopyrimidiniumyl)thio]methyl cephalosporins. J. Med. Chem. 37: $3828 \sim 3833,1994$

10) Murakami, K.; M. Ohashi, A. Matsunaga, I. Yamamoto, A. TOMiguchi \& H. Nohira: Optical resolution by preferential crystallization of 2-(3,4carbonyl dioxyphenyl)-2-(phthalimidooxy)acetic acid. Bull. Chem. Soc. Jpn. 65: 3288 3293, 1992

11) KIM, Y.-Z.; H. S. OH, J.-H. Yeo, J.-C. Lim, W.-S. KIM, C.-S. Bang \& H. J. Yim: Eur. Pat. Appl. EP397,511, 1991; Chem. Abstr. 114: 228626s, 1991 